\title{
Delimiting lipids
}

This themed issue features a collection of articles that explore long-standing questions in the study of lipids and membranes.

A s key structural components of the cell, membranes compartmentalize distinct functions and provide a platform for communication, including receptor-mediated signal transduction, selective transport of molecules, and cell-cell recognition. In this issue, we bring together several Reviews, Perspectives, and Articles that span the varied functions of membranes and their component lipids and proteins, as well as collectively follow historical threads of biochemical and biophysical frameworks from which answers have come from studying the basic building blocks of life.

The study of lipids and membranes began with the recognition of cellular compartmentalization through the use of the first microscopes, yet the fluid mosaic model that describes the organization of cellular membranes was proposed less than 50 years ago. Around that time, organelles could be distinguished by cell fractionation, and biochemical reconstitution studies played a large part in understanding temporal processes such as membrane traffic. The ability to follow dynamic processes in real time has also benefited greatly from live-cell imaging techniques such as photobleaching and photoactivation and super-resolution imaging. However, the molecular details of how membrane structures are built and those that underlie the distinctions between cellular compartments are still sparse, owing in no small part to the partially hydrophobic surfaces and the flexibility and instability of membranes.

Cellular membranes generally take the form of a phospholipid bilayer with two lipid leaflets. The lipids themselves can laterally sort into nanodomains of distinct order and packing that affect fluidity and that serve as specialized signaling platforms. An asymmetric distribution of lipids across the bilayer-for instance with charged lipids being enriched in the cytosolic leaflet-is actively managed by flippases, floppases, and scramblases. This asymmetry has structural and functional consequences, including for communication across membranes, and loss of asymmetry can lead to cell death. Doktorova, Symons and Levental highlight recent work aiming to define membrane composition and organization and pose questions about the origins of membrane asymmetry, the coupling and regulation of the two bilayer halves, and the roles of cholesterol, an abundant membrane lipid, including whether it is enriched in the inner or outer leaflet.

Lipids form a platform for membrane protein function but can also have bioactivity themselves, as seen, for example, with the role of cholesterol in the Hedgehog (Hh) system during development. Radhakrishnan, Rohatgi, and Sebold propose that the Hh receptor PTCH acts as a transporter bringing the cholesterol signal to its transmembrane protein effector SMO. The model suggests that PTCH limits the pool of accessible membrane cholesterol, highlighting the importance of cholesterol distribution. Structural biology has proven to be an invaluable biophysical tool in this system. For instance, $\mathrm{Li}$ and colleagues report cryo-EM findings showing that SMO itself has a sterol tunnel through which cholesterol is activated for downstream signaling.

Structural biology has also illuminated mechanisms of lipid and membrane action on ion-channel gating. Baenziger and Thompson focus on four ion channel families for which structural studies have defined mechanisms of lipid action, including how channel gating is altered by binding of lipids and the membrane itself. Where ion channels have proven difficult to study biochemically, fixed snapshots of channels in various states of activation can be used to construct activation mechanisms. Catterall, Wisedchaisri, and Zheng highlight this approach to reveal the full conformational cycle of a bacterial voltage-gated sodium channel as it transitions from resting to activated-open to inactivated-closed states.

Chorev and Robinson make a strong case for the advantage of membrane mimetics when solving membrane protein structures. With the realization that detergents can alter the protein oligomeric state, destabilize the protein, and encourage non-native lipid interactions, the state of the art has moved to more native-like lipid environments. The evolution of phospholipid bilayers and detergent-free mimetics such as liposomes, vesicles, and nanodiscs has followed a trajectory similar to those of advances in structure determination and mass spectrometry, allowing new insights into regulation and function of membrane proteins.

Advances in structural biology promise to further illuminate the activation of complex membrane proteins such as G-protein-coupled receptors (GPCRs) and ion channels, but classical chemical biology approaches are foundational. In this issue, Javitch and colleagues employ an enzymatic activation mechanism to expose a cryptic tethered agonist in the latrophilin receptor ADGRL3 to show how this GPCR is activated and that it couples to specific G-protein subtypes.

The tools of the chemical biologist have also come to the fore in studying lipids and membranes. Identifying signaling lipids that can act as second messengers and their regulators is an important task ideal for the chemical biologist. For instance, Birsoy and colleagues identified metabolic modifiers of lipid peroxidation including a radical-trapping antioxidant that inhibits lipid peroxidation. Also in this issue, Hamachi and colleagues used biorthogonal labeling to image phosphatidylcholines and their inter-organelle transport in live cells. This approach helped address a long-standing question about the origins of autophagosome membranes. At the membrane level, Goun and colleagues developed a bioluminescent probe to monitor mitochondrial membrane potential in vitro and in mice.

Drug activity for intracellular targets depends heavily on how compounds interact with and permeate membranes. Tan and colleagues argue the need for more diverse chemical libraries and tools to assess permeability and accumulation in Gram-negative bacteria, which are surrounded by a thin peptidoglycan cell wall and an outer membrane containing lipopolysaccharide. Measuring kinetics of accumulation toward understanding the balance between efflux and uptake will aid in finding new effective antibiotics against this challenging bacterial class.

For many aspects of membrane structure and function, chemical biology blends together classical biochemical and structural approaches with emerging techniques for manipulation and molecular-level analysis, promising to provide new insights into membrane and lipid structure and function and the means to manipulate these to modify cell function and treat disease.

Published online: 16 November 2020 https://doi.org/10.1038/s41589-020-00701-6 